\title{
Prémio Gulbenkian 1998 Sistemas Fotocrómicos em Memórias Ópticas. Um olhar sobre a Química como Fonte de Informação
}

\author{
FER N A N O PIN A *
}

\begin{abstract}
Neste trabalho são exploradas as relações entre a Química e a Informação. Descrevem-se ciclos para escrever-ler-apagar baseados na fotoquímica dos sais sintéticos de Flavilio. Discute-se o uso de tais ciclos como modelos na construcão de memórias ópticas, sejam estas permanentes ou temporárias. Mostra-se como se podem conceber redes que permitem passar de uma forma a outra do sal sintético de flavílio através de caminhos diferenciados. Com base em impulsos de $\mathrm{pH}$ ou de luz utilizase o conceito de portas lógicas e definem-se diversas funções.

Introduz-se a noção de perceptron e de redes neuronais baseadas em processos fotoquímicos.
\end{abstract}

one may let one's imagination be carried by the magic of the word and pulled by the evocative and stimulating power of the concept.

Jean-Marie Lehn

\section{FOTÃO "QUANTUM" DE \\ ENERGIA E "BIT" DE INFORMAÇÃO}

Um fotão é um quantum de energia sendo essa sua propriedade explorada pela natureza no processo de Fotossíntese, e pelo Homem em todos os sistemas cujo objectivo é a conversão de energia solar. No entanto um fotão é também um bit de informação, usado pela natureza nos processos de visão, Esquema 1. Estudar a utilização da luz nesta área é um dos objectivos últimos deste estudo.

De um modo geral, este trabalho lida com a geração, transferência, processamento, conversão e detecção de sinais. Todos estes temas se integram num novo ramo da Química

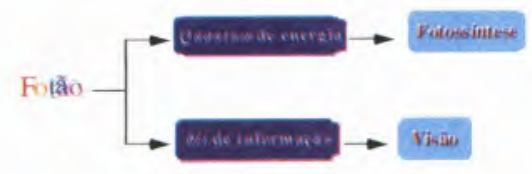

que por proposta de J. M-Lehn, Prémio Nobel da Química em 1987, se pode designar Semioquímica.[1]

A abordagem da química tem seguido uma metodologia que consiste em descer do complexo para o simples, aquilo a que se tem designado por aproximação "top down". A bioquímica é um exemplo flagrante desta estratégia. No entanto é possível conceber uma abordagem diferente onde se sobe do simples para o complexo, a denominada aproximação "bottom up". Nesta estratégia elementos simples e bem caracterizados são adicionados (de preferência auto-ordenados) de modo a dar origem a estruturas cada vez mais complexas, muitas vezes exibindo novas propriedades.

Os componentes dos computadores actuais são construídos por processos litográficos, isto é com base na aproximação "top down". No entanto esta aproximação está a chegar aos seus limites intrínsecos. Uma condição necessária para uma futura miniaturização de modo a aumentar a potência do processamento da informação seria a construção de componentes ao nível molecular usando a aproximação "bottom up", Esquema 2. [1-3]

Curiosamente a possibilidade do computador do futuro vir a operar em solução em vez de no estado sólido, deve também ser posta em consideração. [4] Neste enquadramento o planeamento e construção de sistemas ao nível molecular capazes de

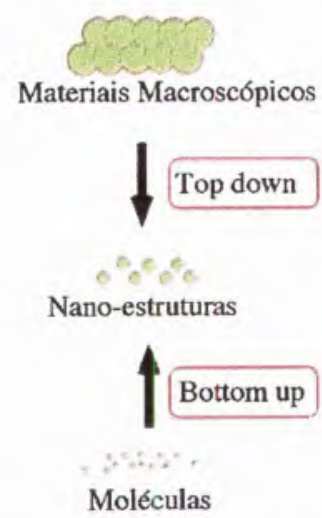

Esquema 2 existir em diferentes formas que podem ser interconvertíveis por estímulos externos é um tema de grande interesse não só do ponto de vista da ciência fundamental como das aplicações inovadoras. [1-8]

Na perspectiva da utilização da Fotoquímica como ferramenta de informação, uma ideia muito simples seria utilizar uma molécula que tenha dois estados diferentes interconvertíveis pela acção da luz, tal como se ilustra no esquema 3. Uma molécula possuindo tais propriedades poderia funcionar como as memórias dos computadores, mas ocupando um espaço muito menor, com as óbvias vantagens.

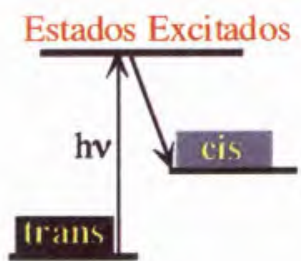

\begin{tabular}{c|c} 
Impulso $(\mathrm{h} v)$ & Estado \\
\hline Não & 0 \\
\hline Sim & 1
\end{tabular}

Esquema 3

Em princípio qualquer reacção fotoquímica é uma memória potencial porque na ausência de luz não reage, estado $\underline{0}$ do sistema, e sob a acção da luz dá origem a um produto, estado 1 do sistema. Trata-se para todos os efeitos de uma escrita digital feita na base dois. Uma isomerização trans para cis é um exemplo desse tipo de reacções fotoquímicas e podia em princípio ser utilizada para escrever por intermédio da luz. A questão é no entanto complexa porque em geral uma reaç̧ão fotocrómica reverte espontaneamente para o estado inicial conferindo instabilidade ao sinal, alínea a), esquema 4. No caso presente o cis formado fotoquimicamente reverteria para o trans. Em 
certos compostos existem barreiras cinéticas e o sistema não reverte termicamente. No entanto para ser lido é necessário que o produto fotoquímico absorva luz. No exemplo anterior por irradiação do cis seria formado o foto-produto trans, alínea b). É este o fenómeno que se pode observar nos conhecidos azobenzenos e noutros compostos análogos. Neste caso o sistema apagaria enquanto era lido.

A solução encontrada para que o sistema escreva e não apague, desvia o produto fotoquímico do equilíbrio trans-cis. Tal pode ser feito utilizando, por exemplo, uma reacção de transferência de protões ou electrões, a que designamos trancar, alínea c). Nesse caso forma-se um produto $Z$ que deve ser estável térmica e fotoquimicamente. Uma vez lido $\mathrm{Z}$, a fase seguinte consiste em apagar. Para tal basta que se proceda à reacção inversa do processo de trancar, destrancar, obter de novo o produto fotoquímico (cis no exemplo) e apagá-lo usando as alínea a) ou b) do Esquema 4.

Sistemas deste tipo podem ser observados nos sais sintéticos de flavílio. Estas moléculas possuem a mesma unidade química básica das antocianinas, que são as moléculas responsáveis pela maioria dos azuis e vermelhos das flores e frutos. Porém, nem todos os sais sintéticos de flavílio são apropriados para aplicar a metodologia referida na alínea c) do esquema 4. Para esse efeito é pelo menos necessário a existência de uma barreira cinética que dificulte a passagem térmica do cis ao trans. Até ao momento só encontrámos a referida barreira nos derivados substituídos na posição $4^{\prime}\left(\mathrm{X}=\mathrm{OCH}_{3}, \mathrm{OH}\right.$ e $\left.\mathrm{CH}_{3}\right)$.<smiles></smiles>
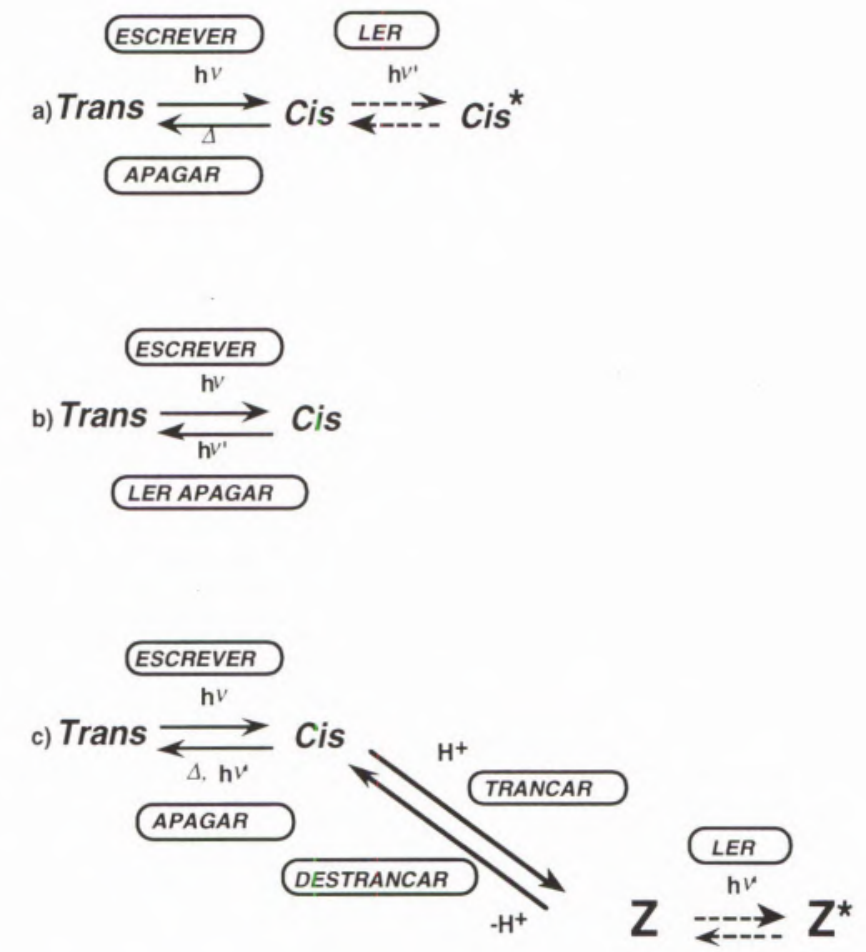

Esquema 4

\section{SAIS SINTÉTICOS DE FLÁVILIO E ANTOCIANINAS}

As antocianinas e os flavílios sintéticos dão origem a 5 espécies maioritárias cujas fracções molares dependem do $\mathrm{pH}$ e do facto do sistema estar ou não no seu equilíbrio termodinâ- mico. A espécie termodinamicamente favorável a valores de $\mathrm{pH}$ muito ácidos é o catião flavílio que nas antocianinas é vermelho e cuja cor nos sais sintéticos de flavílio depende dos grupos substituintes. [9]

À medida que o pH aumenta o catião flavílio desaparece e em sua

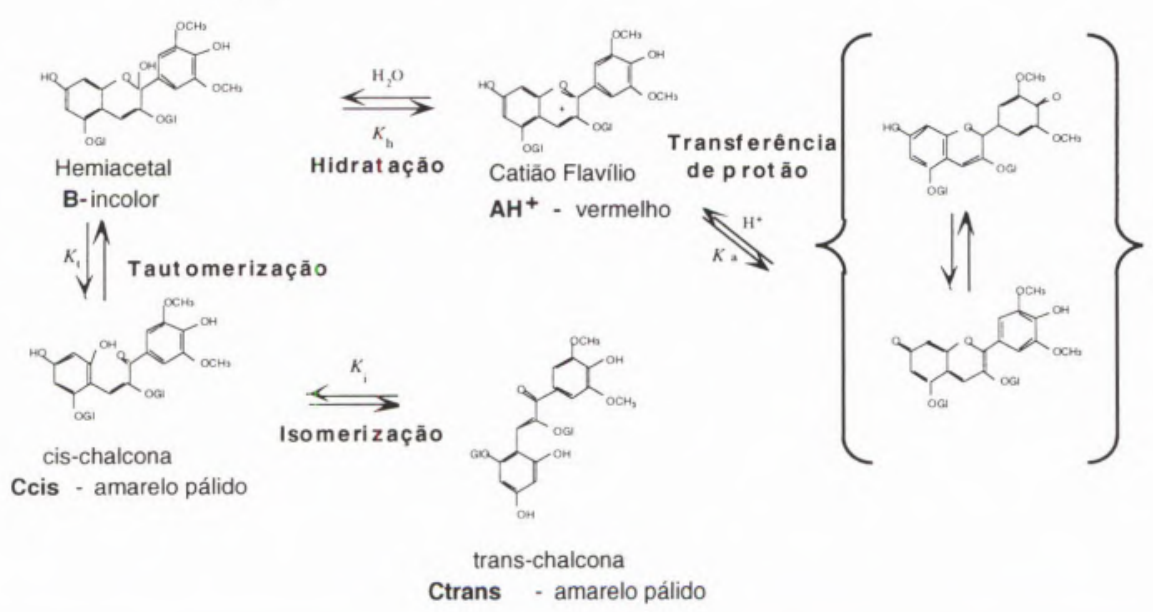

Esquema 5 
substituição formam-se os restantes compostos em quantidades relativas que dependem dos substituintes. No entanto o equilíbrio final não é imediatamente atingido, porque as velocidades dos diversos processos são muito diferentes entre si. Analisemos este comportamento no Esquema 5 que corresponde a uma das antocianinas mais comuns, a Malvina (Malvidina-3,5-diglucósido). Uma solução a pH 1 é de cor vermelha devido ao facto da forma estável a este valor de $\mathrm{pH}$ ser o catião Flavílio. Se adicionarmos base à solução vermelha de modo a atingir um valor de $\mathrm{pH}$ menos ácido, 4 por exemplo, a primeira observação é a verificação da formação imediata de uma cor azul intensa, devido à conversão do catião flavílio na base quinoidal. Esta reaç̧ão é muito rápida (micro segundos) porque se trata de uma simples transferência de protão. No entanto a cor azul não se mantêm e vai desaparecendo ao longo do tempo. Se analisarmos cuidadosamente esse processo verificamos que ocorre em duas etapas distintas: i) uma primeira fase, que dura alguns minutos, ii) uma segunda fase que se completa em algumas horas. O desaparecimento do cor azul na primeira fase é muito mais acentuado do que na segunda. O processo i) foi interpretado considerando um mecanismo de desaparecimento da cor azul (base quinoidal) através do catião flavílio para dar hemiacetal em equilíbrio com cischalcona. Por outras palavras i) corresponde à formação de um pseudoequílibrio entre as formas $\mathrm{A}, \mathrm{AH}^{+}, \mathrm{B}$ e $\mathrm{C}_{\text {cis }}$. Finalmente o equilíbrio final corresponde à formação de uma pequena quantidade de trans- chalcona. Tal comportamento resulta do facto da isomerização cis-trans ser de todos os processos o mais lento. Nesta fase as cinco espécies referidas no Esquema 4 entram no seu equilíbrio termodinâmico. De notar que a pH 4 o hemiacetal é a espécie mais estável. Na Figura 1 (esquerda) mostramos a distribuição das fracções molares da Malvina, logo após o salto de $\mathrm{pH}$, isto é quando somente $\mathrm{AH}^{+}$e $\mathrm{A}$ estão em pseudo-equilíbrio
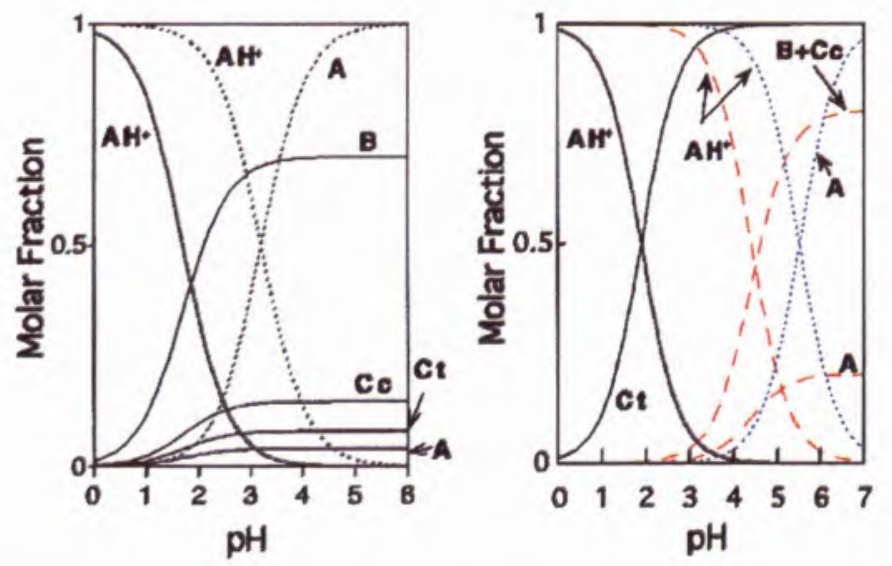

Fig 1 - Distribuição das fracções molares dos compostos Malvina (esquerda) e 4'-Hidroxiflavilio (direita) em função do $\mathrm{pH}$. A cheio a distribuição molar do equilibrio termodimâmico; a tracejado o pseudo-equilibrio entre $\mathrm{AH}^{+} \rightleftarrows \mathrm{A} \rightleftarrows \mathrm{B} \rightleftarrows \mathrm{C}_{\text {cis }}$ (ignorado na Malvina); a pontilhado o pseudo-equilibrio obtido imediatamente após o salto de $\mathrm{pH}$.

e no estado final quando todas as espécies atingiram o equilíbrio termodinâmico.

\section{SAIS SINTÉTICOS DE FLÁVILIO COMO MODELOS DE MEMÓRIAS ÓPTICAS}

Tal como se mostra na Figura 1, e ao contrário das antocianinas, soluções estabilizadas no escuro de muitos dos sais sintéticos de flavílio que temos estudado apresentam um equilíbrio entre o catião flavílio, dominante em meio ácido, e a transchalcona, maioritária em meio neutro ou moderadamente ácido. Esta última espécie é reactiva fotoquímicamente dando origem a uma isomerização trans-cis bastante eficiente. Além disso a cis- chalcona está em equilíbrio com o hemiacetal que por sua vez equilibra com o catião flavílio através de uma reacção que depende do $\mathrm{pH}$. Estão assim criadas as condições enunciadas no Esquema 4 alínea c).

No esquema 6 mostramos como pode o composto $4^{\prime}$-metoxiflavílio ser usado como memória óptica

O ciclo tem início na espécie trans- chalcona que por absorção de luz produz o isómero cis- chalcona. Devido à existência da barreira cinética o sistema não reverte para o estado inicial. No entanto não pode ser lido nesta fase porque nesse caso a absorção de luz pela chalcona cis daria como produto fotoquímico a chalcona trans, e cair-se-ia numa situação de apagar enquanto se lê. Nesta fase é necessário introduzir o passo trancar acidificando a solução de modo a produzir a espécie catião flavílio que é muito estável seja térmica seja fotoquimicamente. Com a introdução deste passo pode ler-se o sistema sem o apagar. Para apagar vai ser necessário levar a efeito o passo de destrancar, no exemplo do esquema 6 adicionando uma base, afim de obter de novo a cis -chalcona que permite reverter para a forma trans seja por irradiação seja por aquecimento a $60{ }^{\circ} \mathrm{C}$.

Um aspecto importante é a possibilidade deste sistema se auto-tran-

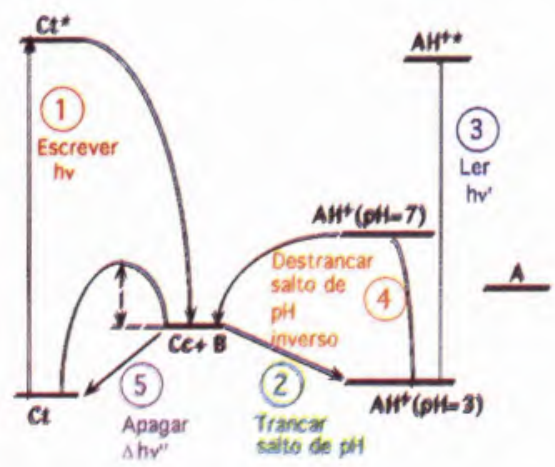

Esquema 6 
car. No caso anterior é possível obter a forma trans- chalcona, por exemplo a $\mathrm{pH}$ 3, escrever com a luz obtendo a cis- chalcona que a este $\mathrm{pH}$ se transforma espontaneamente em catião flavílio $\mathrm{AH}^{+}$que pode ser lido sem apagar. Para apagar basta aquecer a solução a $60{ }^{\circ} \mathrm{C}$.

\section{A LÓGICA MATEMÁTICA E A QUÍMICA}

Um dos aspectos mais fascinantes deste olhar sobre a Química é a possibilidade de associar esta à lógica matemática. Portas lógicas são comutadores cujo sinal de saída $(0,1)$ depende do sinal de entrada $(0,1)$. É um facto aceite que a computação ao nível molecular só pode ser possível quando as portas lógicas estiverem acessíveis. [10]

Afim de obter portas lógicas considera-se, seja um salto de $\mathrm{pH}$, seja um relâmpago de luz, ou mesmo a coordenação de um metal. Estes impulsos dão origem a transformações Químicas, isto é, produzem novas espécies, que por seu lado possuem propriedades diferentes daquelas de partida. A situação pode ser resumida deste modo: as entradas (input) são os impulsos e a saída (output) uma propriedade da espécie química formada, que pode ser uma absorção ou uma emissão de luz, por exemplo. Este tipo de lógica foi por

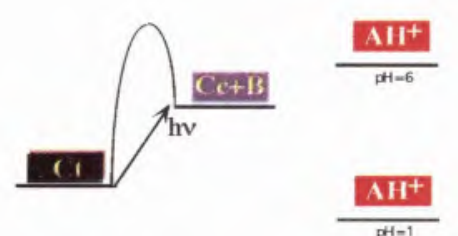

\begin{tabular}{|c|c|c|}
\multicolumn{3}{|c}{ AND } \\
\hline $\mathrm{pH}$ jump & (tw) & State \\
\hline 1 & 1 & 1 \\
\hline 1 & 0 & 0 \\
\hline 0 & 1 & 0 \\
\hline 0 & 0 & 0 \\
\hline
\end{tabular}

Esquema 7 - Representação de uma porta lógica AND [11,12] nós descrito no composto 4'-metoxiflavílio, Esquema 7. [11,12]

Neste esquema o impulso de luz é efectuado com um relâmpago que excita a forma trans-chalcona a $\mathrm{pH}=4.0$; o impulso de $\mathrm{pH}$ é obtido pela adição de ácido através de uma seringa de modo a obter um $\mathrm{pH}$ final de 1. A saída corresponde à observação da formação do catião flavílio seguindo a sua absorção ou a sua emissão de fluorescência. Quando se efectua um salto de $\mathrm{pH} 4$ para $\mathrm{pH} 1$ (entrada 1,0$)$ não se obtém nenhuma formação de catião flavílio devido à existência de uma elevada barreira cinética. O mesmo se passa quando se irradia a trans- chalcona a pH 4 (entrada 0,1$)$ porque o sistema pára no ções, pode ser introduzida utilizando o composto 4'-hidroxiflavílio em vez do 4'-metoxiflavílio. [13,14] No primeiro existe a possibilidade de formação de espécies ionizadas permitindo estender o estudo ao meio básico. $\mathrm{A}$ pH=12 a reacção de formação da trans- chalcona ionizada a partir da cis- chalcona ionizada é lenta, mas pode ser acelerada pela acção da luz, que todavia não tem nenhum efeito na reacção inversa da trans para a cis. Com base nestas propriedades foi possível definir um ciclo em que se podem conceber memórias permanentes e memórias temporárias, Esquema 8. A analogia com o nosso cérebro é por demais evidente pese embora a simplicidade e ingenuidade do modelo.

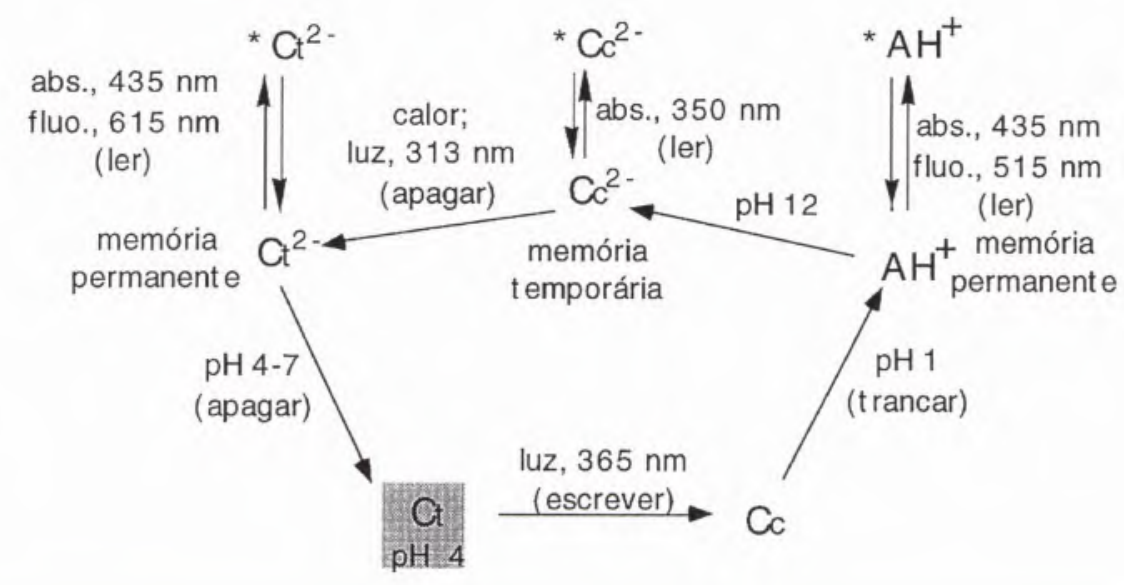

\section{Esquema 8}

equilíbrio cis- chalcona/hemiacetal. Na ausência dos dois impulsos (entrada 0,0$)$ também não se forma catião flavílio (saída 0). Somente quando os dois impulsos estão presentes se verifica formação deste catião. Como se pode concluir da tabela do Esquema 7 trata-se de uma porta lógica que corresponde à função AND.

\section{MEMÓRIAS PERMANENTES E TEMPORÁRIAS}

Uma componente de complexidade, e por consequência uma maior possibilidade de exploração de fun-

\section{MODELOS NEURONAIS}

No exemplo seguinte desenvolve-se a ideia de partir de uma das forma dos sais sintéticos de flavílio e obter uma outra. Esse percurso pode ser efectuado de diversos modos, combinando saltos de $\mathrm{pH}$ com impulsos de luz. De certo modo existe uma vaga analogia com o nosso cérebro, apesar da simplicidade e primitivismo do conceito, Esquema 9.

Tal como na actividade neuronal, são muitas as vias possíveis de ser seguidas, e a morte de uns tantos neurónios não impede que a informação circule. 


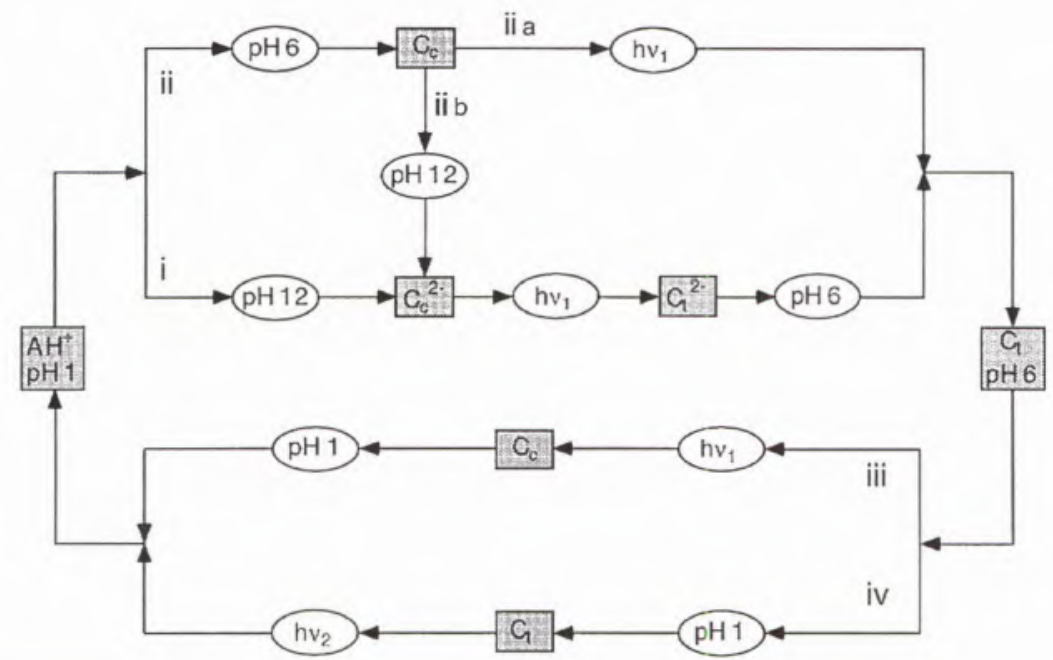

Esquema 9

\section{O CONCEITO DE PERCETRON}

Nos últimos meses temos vindo a fantasiar em sistemas que sejam capazes de apresentar um comportamento do tipo neurónio, mesmo que minimal. Se bem que a bioquímica dos neurónios seja muito complexa, as operações lógicas que ele produz são por contraste bastante simples. [15] O neurónio tem múltiplas ligações de entrada, e de um certo modo soma todos os sinais que chegam através dessas entradas. Se a soma dos sinais de entrada está abaixo de um valor limiar, o neurónio fica desligado e não dá sinal. Se pelo contrário a soma dos sinais de entrada ultrapassa a energia limiar então o neurónio liga e emite um sinal. O neurónio volta ao estado de repouso após um determinado tempo.

Um neurónio artificial com estas características designa-se perceptron elementar. Os sucessivos impulsos que chegam à forma $\mathrm{Ct}$ são somados (de uma forma que depende do $\mathrm{pH}$ ) e podem dar origem ao sinal final. Podemos desligar o nosso sistema usando um pH menos ácido e ligá-lo tornando o $\mathrm{pH}$ mais ácido.

No exemplo seguinte apresentamos um sistema (foto)químico que se comporta como um dispositivo com valor limiar e que é capaz de efectuar uma porta lógica do tipo XOR (OR exclusivo), além de permitir integrar o efeito de dois sinais de entrada num só sinal de saída.

A ideia é muito simples. Na mesma solução que contém o sal 4 '-metoxiflavílio junta-se o composto de coordenação hexacianocobaltato (III), $\left[\mathrm{Co}(\mathrm{CN})_{6}\right]^{3-}$. A luz passa a ser partilhada entre a sal de flavílio e o composto de coordenação, ver Figura 2. A absorção de luz por este último composto dá origem à substituição de uma molécula de cianeto por água com o consequente aumento do $\mathrm{pH}$ da solução, ver eq.(1).

$$
\begin{aligned}
& {\left[\mathrm{Co}(\mathrm{CN})_{6}\right]^{3-}+\mathrm{H}_{3} \mathrm{O}^{+}-\stackrel{\mathrm{hv}}{\rightarrow} \rightarrow} \\
& \rightarrow\left[\mathrm{Co}(\mathrm{CN})_{5}\left(\mathrm{H}_{2} \mathrm{O}\right)\right]^{2-}+\mathrm{HCN}
\end{aligned}
$$

Quando uma solução ácida a $\mathrm{pH}=3.6$ contendo a forma trans-chalcona $(\mathrm{Ct})$ e $\left[\mathrm{Co}(\mathrm{CN})_{6}\right]^{3-}$ é irradiada a $365 \mathrm{~nm}$, a maioria da luz incidente é absorvida pela $\mathrm{Ct}$ dando origem ao

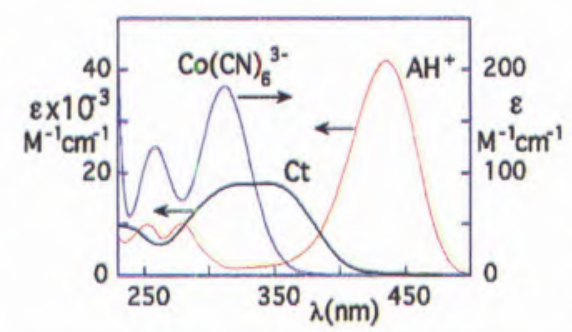

Fig. 2 - Espectros de absorção dos componentes do sistema XOR produto Cc. De início o pH da solução é suficientemente ácido para permitir que Cc se transforme em $\mathrm{AH}^{+}$, obtendo-se o sinal. Com a continuação da irradiação a quantidade de luz que a forma $\mathrm{Ct}$ vai absorvendo tende a diminuir ( $\mathrm{Ct}$ vai sendo consumido) e cada vez mais luz passa a ser absorvida pelo composto de cobalto, com o consequente aumento de $\mathrm{pH}$. Este aumento de $\mathrm{pH}$ faz com que o flavílio inicialmente formado se vá transformando em Cc (em equilíbrio com B) e dependendo do tempo e das condições experimentais acabar mesmo por reverter para $\mathrm{Ct}$.

Na Figura 3 está representada a variação da aborvância devida à formação da espécie $\mathrm{AH}^{+}$. De acordo com esta figura a formação de $\mathrm{AH}^{+}$passa por um máximo e acaba por quase desaparecer. No canto superior direito da Figura 3 está igualmente representado o resultado da acção de dois impulsos de luz (flash) de igual intensidade. Enquanto que com um impulso se consegue um sinal, com dois impulsos o sinal diminui drasticamente (Esquema 10)

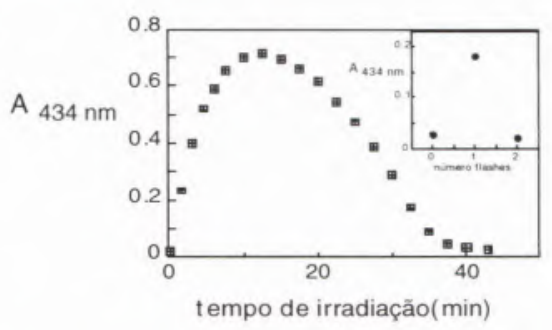

Fig.3 - Variação da absorvância da espécie AH+ com o tempo de irradiação.

\section{PERSPECTIVAS FUTURAS}

Ainda não é claro se estes sistemas irão ter aplicação prática num futuro imediato. Até lá muitos problemas restam por resolver, como sejam; i) a velocidade das reacções, ii) a necessidade de uma enorme estabilidade dos compostos que ainda só parcialmente foi atingida, iii) a 
output

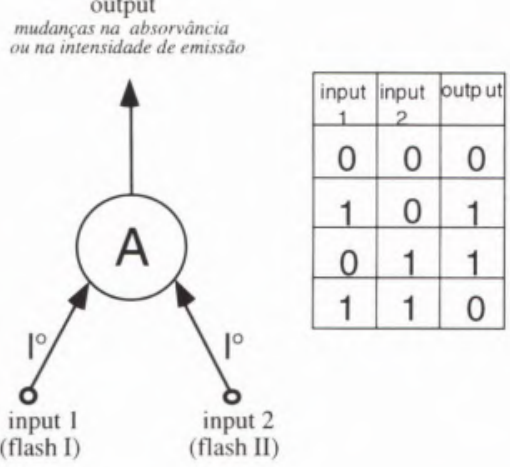

Esquema 10

passagem dos sistemas em solução para matrizes sólidas ou meios organizados. No entanto este tipo de estudos é importante porque estimula o engenho dos Químicos e introduz novos conceitos e novas ideias. Aqui gostariamos de voltar a referir a frase de Jean-Marie Lehn com que iniciámos este trabalho. ...one may let one's imagination be carried by the magic of the word and pulled by the evocative and stimulating power of the concept.

\section{AGRADECIMENTOS}

Este trabalho resulta de uma longa colaboração entre os Prof. V. Balzani e M. Maestri da Universidade de Bologna, Italia e o meu grupo de investigação na UNL. Gostaria de agradecer em particular à Prof. Maria João Melo, à Dr. Ana Roque e ao Prof. Jorge Parola, os pilares que tantas vezes na sombra tornaram possivel que vos apresente este trabalho à luz do dia (ou não estivéssemos no domínio da fotoquímica). Uma última palavra de agradecimento para a Fundação para a Ciência e a Tecnologia que nos tem vindo a financiar e para a Fundação Gulbenkian pelo estímulo que nos concedeu.

\section{* Departamento de Química, Centro de Química Fina e Biotecnologia, Faculdade de Ciências e Tecnologia, Universidade Nova de Lisboa. Quinta da Torre, 2825-114 Monte de Caparica, Portugal-e-mail:fjp@dq.fct.unl.pt}

\section{REFERÊNCIAS}

1. J- M. Lehn "Supramolecular Photochemistry. Concepts and Perspectives" 1995, VCH. Darmstadt. Alemanha.
2. Molecular Electronic Devices, ed. F.L. Carter, R:E: Siatkowsky, H. Woltien, Elsevier, Amsterdam, 1988.

3. D. Rouvray, Chem. Brit. 1998, 34(2), 26.

4. P. Ball, L. Garvin, Nature, 1992, 355, 761; D Bradley, Science, 1993, 259, 890.

5. V. Balzani, F. Scandola, Supramolecular Photochemistry, Ellis Horwood, Chichester, 1991.

6. L.B-, Feringa, W.F. Jager, B. de Lange, Tetrahedron 1993, 49, 8267 .

7. A.P. de Silva, C.P. Mc Coy, Chem. Ind. 1994, 992.

8. L. Fabbrizzi, A. Poggi, Chem. Soc. Rev. 1995, 24, 197

9. M.J. Melo, A. Roque, F. Pina, Boletim da SPQ, $1999 \mathbf{7 4}, 41$

10. A. Credi, V. Balzani, S. J. Langford, J.F. Stoddart, I. Am. Chem. Soc. 1997, 119, 2679.

11. F. Pina, M. J. Melo, M. Maestri, R. Ballardini, V Balzani, J. Am. Chem. Soc. 1997, 119, , 5556.

12. F. Pina, M. Maestri, V. Balzani, J. Chem. Soc Chem.Commun. 1999, 107.

13. F. Pina, A. Roque, M. J. Melo M. Maestri, L. Belladelli, V. Balzani Chem. Eur. J. 1998, 4, 1184-1191.

14. F. Pina, M. J. Melo, A.J. Parola, M. Maestri, V. Balzani, Chem. Eur. J. 1998, 4 2001-2007.

15. H. M. Cartwright "Applications of Artificial Intelligence in Chemistry" Oxford Sicence Publications, 1993.

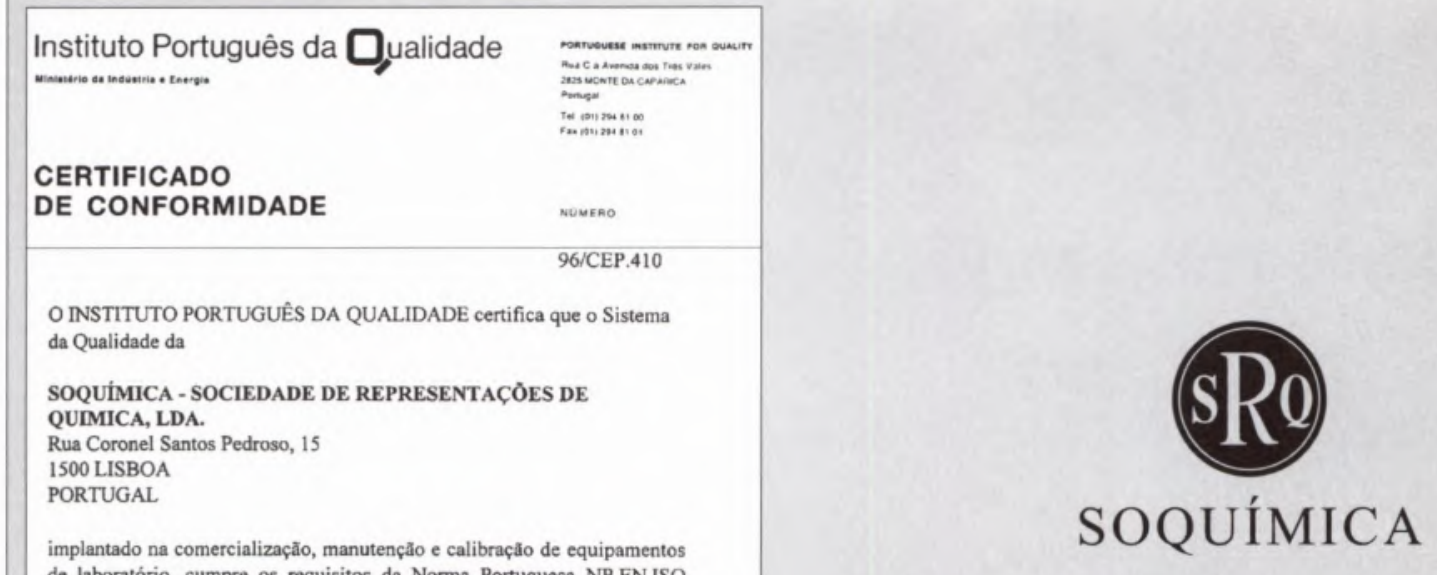

de laboratório, cumpre os requisitos da Norma Portuguesa NP EN ISO 9002 - "Sistemas da Qualidade. Modelo de garantia da qualidade na produçăo, instalaçăo e assistência após venda"

O presente certificado é emitido ao abrigo do Decreto-Lei n. ${ }^{\circ} 234 / 93$ de 2 de Julho, e de acordo com a Directiva CNQ 22 - "Certificação de sistemas da qualidade de empresas. Metodologias e regras gerais".

A presente certificação é válida por um periodo de trés anos, renovável.

Monte de Caparica, 18 de Junho de 1996

\section{Caintidion Sautor}

Sociedade de Representações e Química, Lda.

Rua Coronel Santos Pedroso, 15 • 1500 LISBOA • Tel.: 7165160 • Fax: 7165169 Sede Social: Av. da Liberdade, 220-2 $\bullet 129$ LISBOA CODEX

Rua 5 de Outubro, 269 • 4100 PORTO • Tels.: 6093069 • Fax: 6000834 Email: soquimica@ mail.telepac.pt; Internet: www.soquimica.pt 


\section{אמ

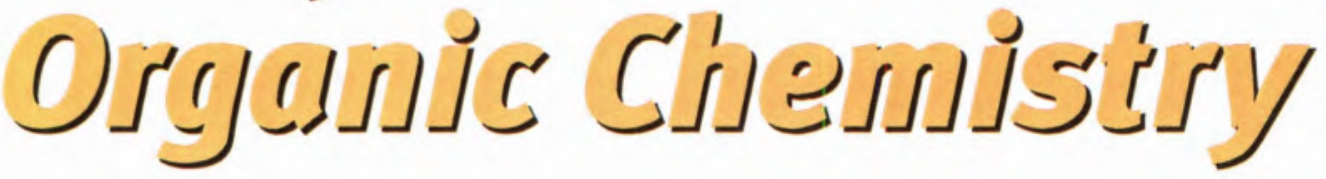

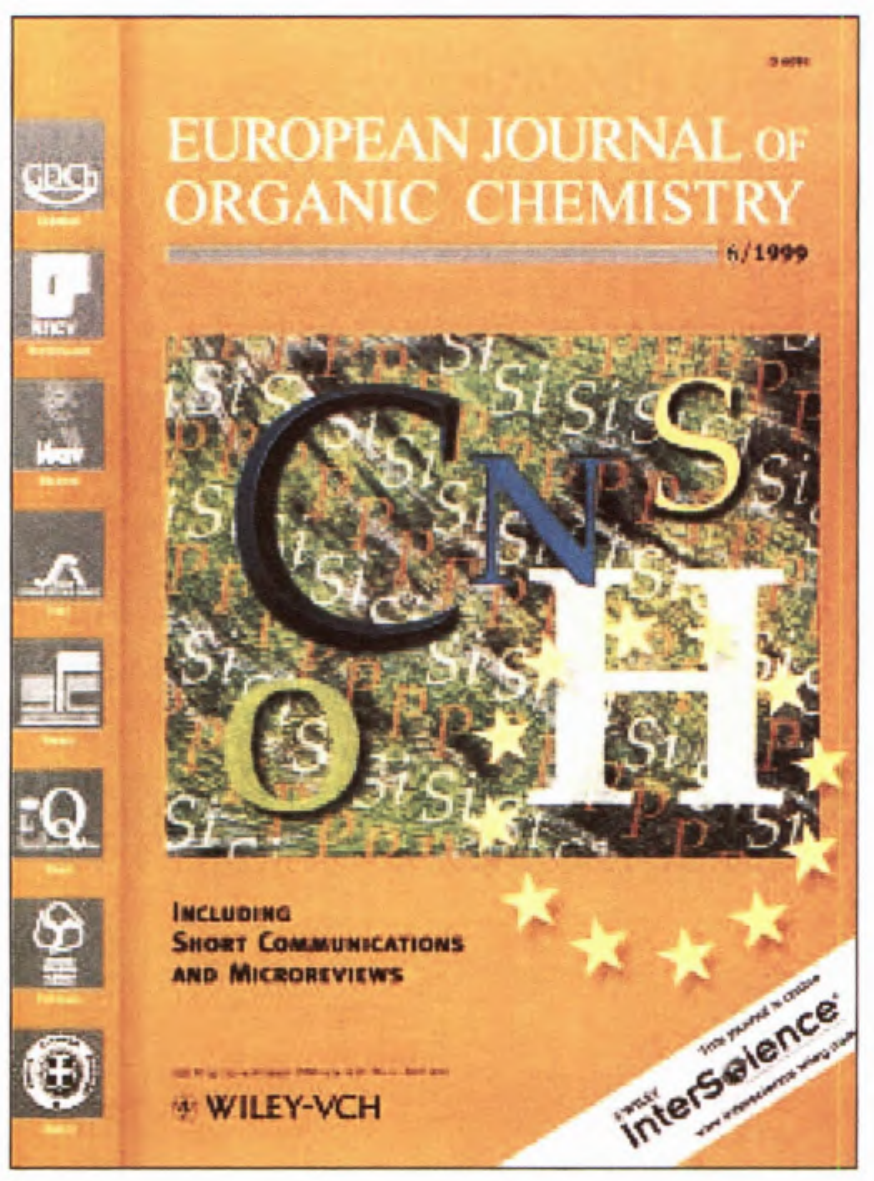

Benefit from:

Full-text available online for subscribers (www.interscience.wiley.com) strong international coverage faster publication times: articles available online weeks before print edition cutting-edge research and information low personal subscription rate for members of supporting societies

doubled publication frequency:

24 issues from 2000

\section{supported and owned by Chemical Societies from}

\section{$B \bullet D \bullet E \bullet F \bullet G R \bullet I \bullet N L \bullet P$}

To order please contact your society or EJIC-EJOC@wiley-vch.de 


\section{Europedn Journal of unorganic Chemistry}

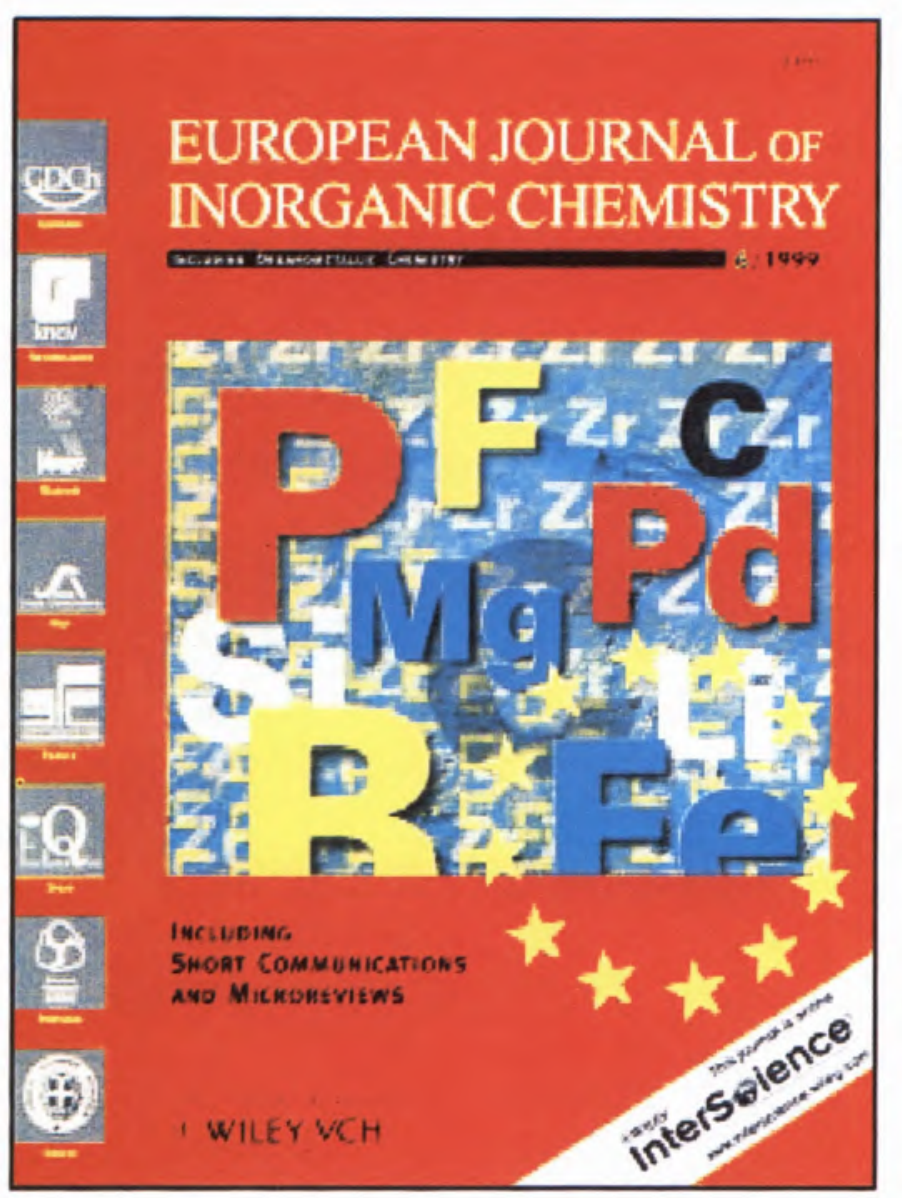

\section{$12 \%$ more pages planned for 2000}

Benefit from:

.... Full-text available online for subscribers

(www.interscience.wiley.com)

.... strong international coverage

.... faster publication times:

articles available online

weeks before print edition

.... cutting-edge research and information

.... low personal subscription rate for members of supporting societies

\section{supported and owned by Chemical Societies from}

\section{$B \bullet D \bullet E \bullet F \bullet G R \bullet I \bullet N L \bullet P$}

To order please contact your society or EJIC-EJOC@wiley-vch.de 


\section{Chemistry -}

A European

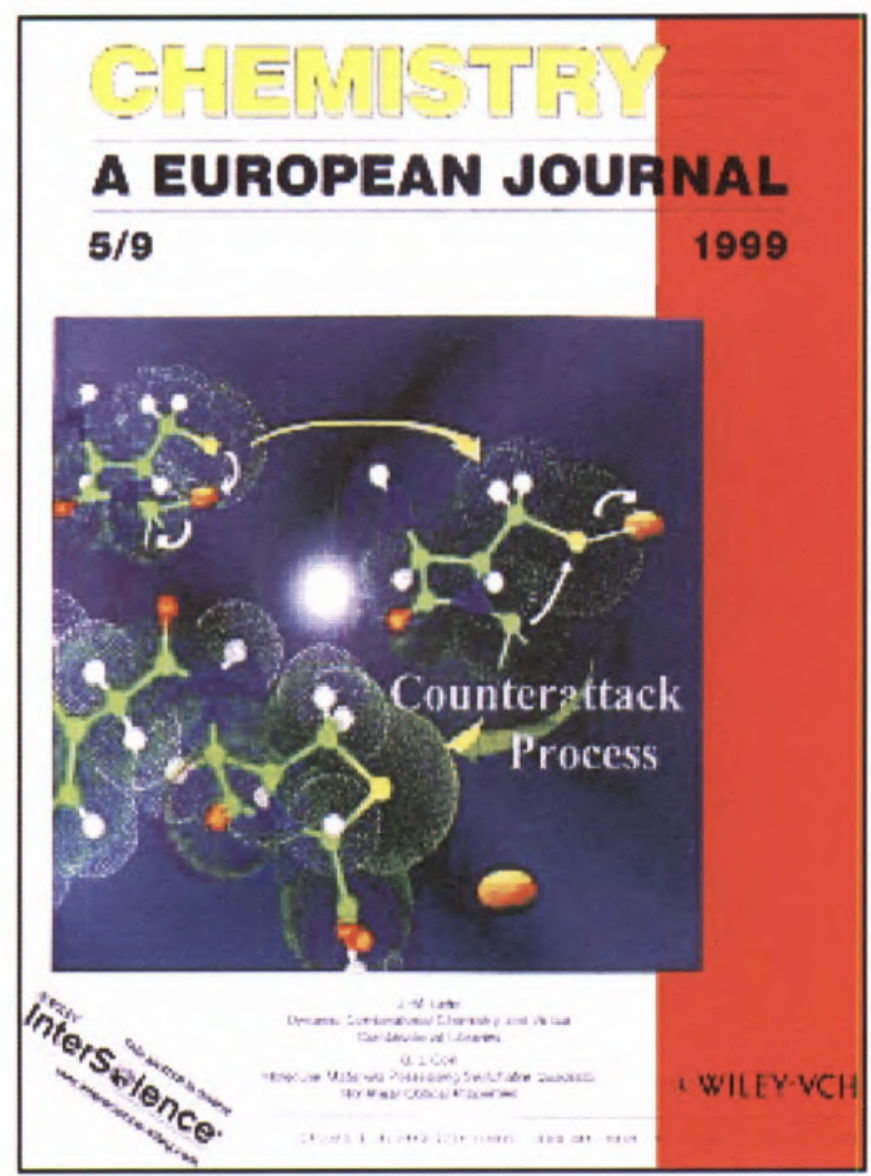

doubled publication frequency: 24 issues from 2000

\section{Journal}

Benefit from:

.... Full-text available online for subscribers

(www.interscience.wiley.com)

.... international authors

.... faster publication times:

articles available online weeks before print edition

.... high-quality full papers

... low personal subscription rate for members of supporting societies

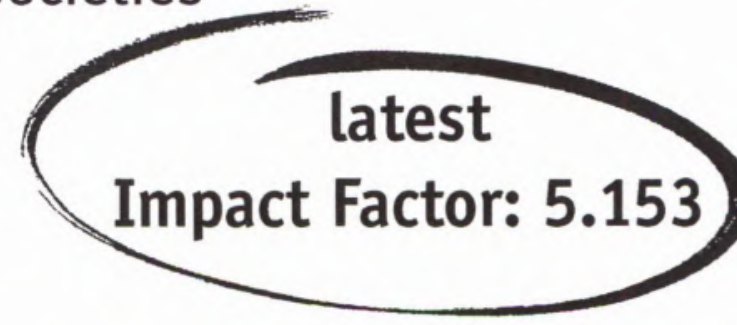

supported and owned by Chemical Societies from $A U \bullet B \bullet C Z \bullet D \bullet E \bullet F \bullet G R \bullet H \bullet I \bullet N L \bullet P \bullet P L \bullet S$

To order please contact your society orCHEMISTRY@wiley-vch.de 